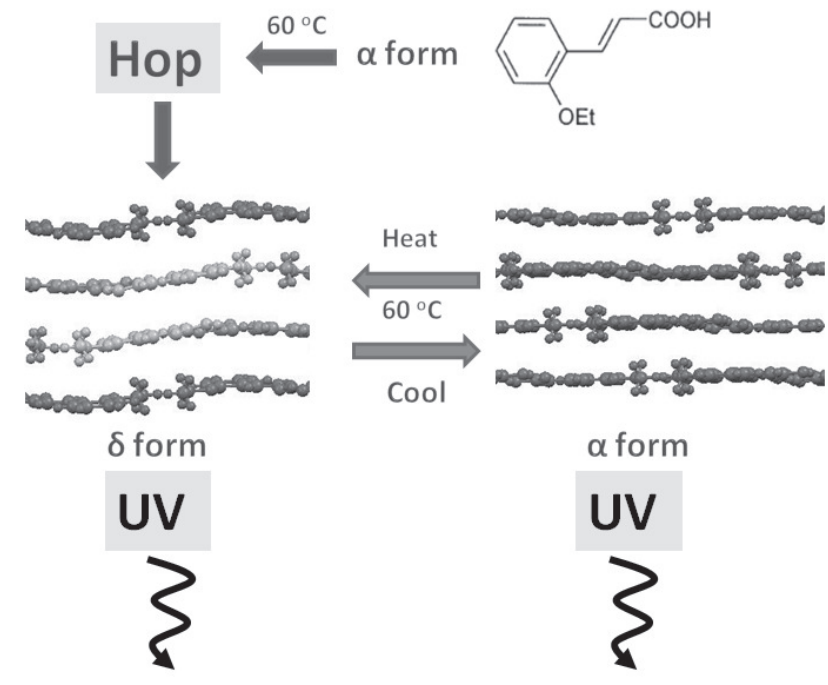

Figure 1. Schematic diagram showing the effect of heating and cooling the $\alpha$ and $\delta$ (or $\alpha^{\prime}$ ) polymorphs of oetca

Keywords: solid-state reaction, photodimerisation, crystal hopping, thermosalient effect, single-crystal-to-single-crystal phase transformation, polymorph

\section{MS33-O4 Identification of the cleavage planes in molecular crystals: topological and energetic aspects}

\author{
Pavel N. Zolotarev ${ }^{1}$, Massimo Moret ${ }^{2}$, Davide M. Proserpio ${ }^{1,3}$
}

1. Samara Center for Theoretical Materials Science, Samara State University, Samara, Russia

2. Dipartimento di Scienza dei Materiali, Università degli Studi di Milano Bicocca, Milano, Italy

3. Dipartimento di Chimica, Università degli Studi di Milano, Milano, Italy

email: paulzolotoff@gmail.com

Thin films of organic semiconductors have a wide application in modern electronic devices. Until now we have used substrate surfaces for organic molecular beam epitaxy (OMBE) [1] provided by b-alanine crystal primary cleavage plane (010). Having a stake in different types and symmetries of substrate surfaces, we searched through the Cambridge Structural Database (release 5.35 Nov 2013) by means of the ToposPro program package [2] modified with a specially tailored procedure that determines possibility of the crystal to cleave along a given surface. This procedure is based on the analysis of the share of intermolecular interaction energy of molecules within the layer compared to the total energy of interaction.

As a result, we have extracted 546 structures of amino acids derivatives with 2-periodic hydrogen bonded network as the most probable candidates for easily cleavable crystals. We discarded the structures of solvates and hydrates as inappropriate in order to guarantee stability to ultra-high vacuum conditions needed for OMBE. For some selected key structures we calculated intermolecular interaction energies by means of the PIXEL method [3] trying to establish the structural features that can lead to the ability of molecular crystals to cleave. In addition, we proposed a quantitative descriptor of intermolecular interaction anisotropy - the $\mathbf{X}$ parameter.

To prove the validity of the proposed scheme we crystallized five crystals and seven co-crystals of amino acids. Unit cell parameters as well as face indexing procedure were carried out on a diffractometer. Subsequently we tested the crystals on the cleavage. For $\beta$-alanine crystals, during the squeezing between two glass plates, secondary cleavage planes along $\{111\}$ faces were found that is in full agreement with our prediction.

The work was supported by the Russian government (Grant 14.B25.31.0005).

\section{References:}

[1] S. Trabattoni, M. Moret, M. Campione, L. Raimondo, A. Sassella Cryst. Growth Des. 2013, 13, 4268.

[2] V.A. Blatov, A.P. Shevchenko, D.M. Proserpio Cryst. Growth Des. 2014, 14, 3576.

[3] A. Gavezzotti J. Phys. Chem. 2002, 106, 4145; A. Gavezzotti J. Phys. Chem. 2003, 107, 2344. 


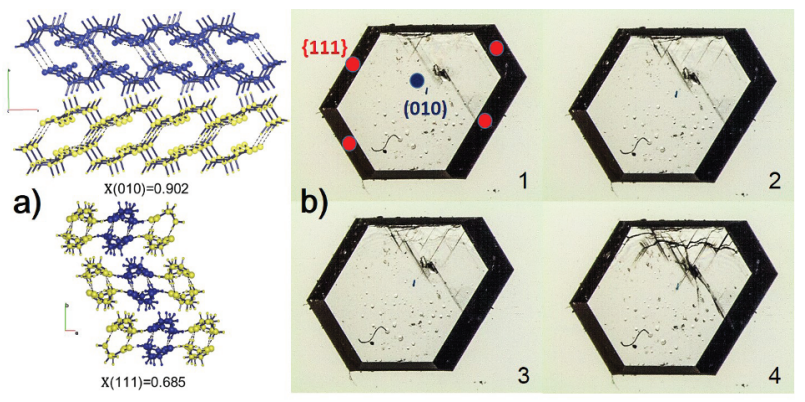

Figure 1. a) Computed values of $X$ parameter for (010) and (111) layers in the structure of $\beta$-alanine; b) Sequential frames obtained during the squeezing of $\beta$-alanine crystal. Appearing secondary cleavage planes are parallel to $\{111\}$.

Keywords: Intermolecular interactions, mechanical properties of organic solids

\section{MS33-O5 Solvent responsive 2D coordination networks: Breathing Materials \\ Gaelle Ramon ${ }^{1}$, Gift Mehlana ${ }^{1}$, Susan Bourne ${ }^{1}$}

1. University of Cape Town

email: gaelle.ramon@uct.ac.za

The development of Metal Organic Frameworks (MOFs) was driven by the chemists' efforts to reproduce the microporosity observed in naturally occurring zeolites. By combining metal nodes and organic spacers, new crystalline compounds were obtained, displaying 3D porous structures and a certain robustness. Initially favoured, the 3D structures showed limitations with respect to some applications such as guest selectivity and separation. These were better achieved by another category of MOFs which were guest-responsive and dynamic: the breathing MOFs. These MOFs afforded added functionalities to that of the robust 3D materials through reversible structural transformations between large and small cavities. In this paper we will be presenting a novel $2 \mathrm{D}$ Breathing $\mathrm{MOF}^{1-2}$ assembled from mononuclear zinc(II) centres and 3-(4-pyridyl)benzoate ditopic linker. The novel material allows for the accommodation of large guest molecules with diameters larger than the original pores of their empty framework. The mechanism of the motion of the dynamic network will also be carefully described.

Keywords: MOF, sorption, crystalline structure 